\title{
Phaethon-Gemind complex by Pan-STARRS
}

Shinsuke Abe

\author{
Institute of Astronomy, National Central University
}

email: shinsuke.avell@gmail.com

\begin{abstract}
The physical nature such as orbital distribution of asteroids is fundamental to understanding how our solar system has been evolved. The connection between Near-Earth Objects (NEOs) and Earth impactors such as meteorites and fireballs are still under debate, since there is no meteorite orbit whose parent NEO was identified. The orbital distribution of NEOs has been investigated by comprehensive sky surveys including Pan-STARRS (The Panoramic Survey Telescope And Rapid Response System). Here we focus on the Phaethon-Gemind complex detected by Pan-STARRS PS1 Prototype Telescope and our follow-up lightcurve observations.
\end{abstract}

